Revista Española de Antropología Americana ISSN: 0556-6533

https://doi.org/10.5209/REAA.63687

\title{
Acceso a internet desigual y heterogéneo en los pueblos indígenas de la Sierra Norte de Puebla (México)
}

\author{
Óscar Ramos Mancilla ${ }^{1}$
}

Recibido: 27 de febrero de 2017 / Aceptado: 20 de agosto de 2018

Resumen. La población indígena utiliza cada vez más las tecnologías de información y comunicación, pero múltiples desventajas y exclusiones provocan que su acceso remarque relaciones de desigualdad. En torno a estas y otras relaciones, la vida comunitaria continúa reorganizándose. En este trabajo se hace un acercamiento sociohistórico al acceso a las TIC por medio de la descripción de lugares y personas vinculadas a las tecnologías digitales en la Sierra Norte de Puebla (México). Se destaca que la conexión a internet, además de ser desigual, se va conformando heterogénea a partir de las interacciones entre las personas y los recursos digitales, donde adquieren importancia las características de las tecnologías, pero aún más el papel de las personas que facilitan el acceso y de las dinámicas locales de los poblados con los que se comparten valores y sentidos de la comunidad.

Palabras clave: acceso a internet; telecentro público; cibercafé; pueblos indígenas.

\section{[en] Unequal and Heterogeneous Connectivity in Indigenous Communities of Sierra Norte de Puebla (Mexico)}

\begin{abstract}
Indigenous people are more and more using information and communication technologies, but multiple disadvantages and exclusions make their access reinforce relations of inequality. Amidst these relations, community life keeps reorganizing itself. This work socio-historically approaches ITC access through the description of places and people linked to digital technologies in Sierra Norte de Puebla (Mexico). It is highlighted that internet connection, besides being unequal, become heterogeneous through the interaction between people and digital resources. Of special importance in that process are the characteristics of technologies, but even more important are the role of people who facilitate access to them and the local dynamics of communities with shared values and community meanings.
\end{abstract}

Keywords: internet access; public telecenter; cybercafé; indigenous peoples.

Sumario. 1. Introducción. 2. Nota metodológica. 3. Lugares de acceso y diversificación de relaciones. 4. Las personas nodos. 5. La constante configuración social del acceso a lo digital. 6. Conclusiones. 7. Referencias.

Cómo citar: Ramos Mancilla, Óscar. 2018. «Acceso a internet desigual y heterogéneo en los pueblos indígenas de la Sierra Norte de Puebla (México)». Revista Española de Antropología Americana 48: $9-27$.

\footnotetext{
1 Grupo de Estudios sobre Culturas Indígenas y Afroamericanas, Universitat de Barcelona. oscar.orm@gmail.com
} 


\section{Introducción}

La conexión a internet en los pueblos indígenas puede considerarse un aspecto tangencial frente a necesidades prioritarias como agua y servicios de salud o el pleno ejercicio de derechos culturales y territoriales. Sin embargo, el aumento de la infraestructura de telecomunicaciones y la disponibilidad de tecnologías digitales en estos poblados invitan a cuestionar las condiciones materiales y los contextos de las conexiones digitales.

Según el Instituto Nacional de Estadística y Geografía y la Asociación de Internet .Mx se estima que en 2016 había unos 65 millones de usuarios de internet en México (AiMx 2016; Inegi 2016), mostrando un aumento significativo frente a años anteriores; por ejemplo, en 2011 se indicaban poco más de 40 millones de usuarios (AiMx 2011). A pesar de dicho incremento, entre las entidades federativas con menores índices de conexión están Puebla, Michoacán, Tlaxcala, Guerrero, Tabasco, Oaxaca y Chiapas, que son precisamente los estados con mayor población indígena y en donde se concentran mayores desigualdades socioeconómicas (Conapo 2015).

Trabajos previos interesados en el acceso a las tecnologías de información y comunicación (TIC) en poblaciones indígenas en México, han señalado varios usos y apropiaciones por jóvenes (Becerra Cortés 2013; Guerra García y Miranda Bojórquez 2010) y por organizaciones y colectivos (Gómez Montt 2005; Sandoval Forero 2013). Con ello exponen un panorama complejo por la diversidad de incursiones a los ambientes digitales, pero carecen de cuestionamientos sobre las posibilidades del acceso a TIC y su vinculación con aquellas conexiones a internet que describen, obviando que estas posibilidades son atravesadas por «políticas de exclusión» (Delgado 2002) y por distintos aspectos locales que derivan en la conformación de accesos diferenciados a la vez que tienen consecuencias diferenciadoras. Otras investigaciones han identificado que la simple posibilidad del acceso a las TIC significa únicamente eso: «la posibilidad del acceso». Para comprender las interacciones entre las personas y las TIC y, de manera más amplia, los procesos sociales vinculados a las conexiones a internet, es necesario especificar otros aspectos; por ello, Eszter Hargittai (2004) indica que, superada la cuestión de la infraestructura, lo que adquiere mayor importancia son otros factores como la educación o el tejido organizativo comunitario; por otro lado, Jenna Burrell (2011) también identifica que el acceso a internet es atravesado por otros filtros, como los rumores que circulan entre las y los jóvenes sobre lo que se puede hacer con y en internet, y que son esas ideas previas las que intervienen en las apropiaciones posteriores.

Así, el objetivo de este artículo con enfoque etnográfico es describir las características del acceso a las tecnologías de información y comunicación, y exponer algunos aspectos en torno a la conectividad en el contexto de los pueblos indígenas de la Sierra Norte de Puebla. Para ello, la noción de campo social (Roseberry 1998) es útil a la hora de observar que las posibilidades de acceso a las TIC y de conexión a internet se van conformando históricamente, que en ellas participan diversos sujetos sociales, que en torno a ellas se despliegan diferentes relaciones sociales e intervienen aspectos locales que son los que terminan moldeando las características específicas de las conexiones digitales en poblaciones indígenas. 


\section{Nota metodológica}

El acercamiento antropológico que se realiza en este artículo aborda el acceso a las TIC como parte de un campo social más amplio que incluye las condiciones materiales y las relaciones sociales en torno a la conexión a internet y, además, las interacciones y expresiones digitales. Se asume que el campo social es el ámbito que permite y en el que se expresan las relaciones sociales -en términos de que el campo permite y al mismo tiempo delimita-, además de que esas relaciones van redefiniendo las posiciones sociales y las agencias entre los sujetos sociales, así como el campo mismo (Roseberry 1998). Así, en la descripción de los lugares de acceso público y de las personas responsables de estos lugares, se puede indicar que las condiciones materiales y las relaciones sociales que generan son producidas por distintas interrelaciones entre agentes y sujetos sociales en torno a los recursos digitales a los que acceden y que van utilizando, además de las propias exploraciones que realizan las personas mientras incorporan las tecnologías digitales en sus actividades.

La información que se expone corresponde, principalmente, al trabajo de campo realizado en los municipios de Ixtepec y Xochitlán de Vicente Suárez entre los años 2012 y 2013, pero se retoman notas y observaciones de diferentes periodos de campo en la Sierra Norte de Puebla desde 2003 con objeto de realizar comparaciones de las situaciones de conexión a internet y un seguimiento de algunos procesos vinculados a las tecnologías digitales. El registro de la información en todos los casos ha sido la observación en campo y entrevistas y conversaciones con diferentes personas, desde usuarios hasta quienes están a cargo de los lugares de acceso público a TIC. El número de estos lugares era reducido, lo que permitió el acercamiento en todos ellos; además fueron espacios de encuentros ocasionales que muchas veces derivaron en pláticas informales. La orientación de las preguntas se dirigió a las posibilidades de acceso a tecnologías digitales y a las maneras que tienen para conectarse a internet, a sus usos, las implicaciones en las relaciones interpersonales, el acceso a redes sociales, también a las actividades diarias y otros asuntos de la vida en los poblados.

La Sierra Norte de Puebla es una demarcación geopolítica compuesta por $63 \mathrm{mu}-$ nicipios de los 217 del Estado de Puebla. El etnólogo Manlio Barbosa Cano (2012) ha propuesto una regionalización ${ }^{2}$ que se corresponde más con los grupos de población que habitan en el territorio y los flujos socioeconómicos; divide esta área en dos según la preponderancia del grupo étnico: nahuas del parteaguas de la Sierra Norte y totonaca de la vertiente del Golfo. La región totonaca colinda con el Estado de Veracruz donde se extiende el Totonacapan y la región Nahua se ubica al sur, en las partes más altas. Además de nahuas y totonacos, también habitan otomíes, tepehuas y población mestiza. Si bien la población indígena está dispersa por toda la Sierra Norte, es en los poblados mestizos donde se ha concentrado la infraestructura de servicios públicos y en consecuencia tienen mayores posibilidades de conexión a internet, pero fuera de estas aglomeraciones urbanas hay otros poblados desde los cuales también se están realizando conexiones, aunque con otras condiciones de infraestructura.

\footnotetext{
2 Divide al Estado de Puebla en diez regiones: Valles Centrales, Nahua, Totonaca, Llanos de San Juan, Atlixco, Izúcar-Chietla, Mixteca-Popoloca, Tepeaca-Tecamachalco-Tehuacán, Iztaccíhuatl-Popocatépetl, Citlaltépetl-Sierra Negra y Tentzo (Barbosa 2012).
} 


\section{Lugares de acceso y diversificación de relaciones}

Aquí se denomina «lugares de acceso público a TIC» a los sitios donde se puede acceder a tecnologías digitales, que pueden derivar en conexión a internet y que están, además, abiertos a todas las personas (aunque, como se menciona más adelante, el hecho de que sean abiertos no implica que todas las personas puedan utilizar las TIC). Para su descripción, estos lugares de acceso público se han divido en tres tipos a partir de la secuencia temporal con que se instalaron en la Sierra Norte. El primero son los telecentros públicos instalados por el gobierno estatal de Puebla; el segundo corresponde a la iniciativa nacional de telecentros llamados Centros Comunitarios de Aprendizaje; y el tercero son los cibercafés, que en años recientes han incrementado su número y son los más utilizados.

\subsection{Telecentros públicos del Estado de Puebla}

Los telecentros propuestos por el gobierno del Estado de Puebla fueron denominados Centros Regionales y se formalizaron en 1998 dentro del Sistema de Información y Comunicación del Estado de Puebla (Sicom) $)^{3}$ con el objetivo de ser

«promotores y prestadores directos de tres servicios específicos: a) Cómputo (acceso a Internet, correo electrónico, software educativo, impresión, venta de diskettes, asistencia técnica); b) Módulo de consulta (materiales en video y audio); c) Sala de usos múltiples, con recepción de la señal Edusat» (Cecavi 2000: 20).

A nivel nacional, fueron, junto con los del Estado de Guanajuato, las primeras exploraciones de una agenda digital que se tomaba como política estatal.

En la Sierra Norte se instalaron, en un inicio, Centros Regionales en las cabeceras municipales de Huauchinango, Zacatlán, Zacapoaxtla y Teziutlán y después, en años diferentes y sucesivamente, los de Huehuetla, Olintla, Atlequizayan, Jonotla, Pantepec y Cuetzalan del Progreso ${ }^{4}$. Los lugares en donde fueron instalados los primeros Centros Regionales corresponden a las cabeceras de distrito y de jurisdicciones, ya que se pretendía que el acceso a las TIC apoyara a la educación formal, específicamente desde las Coordinaciones Regionales de Desarrollo Educativo de la Secretaría de Educación Pública y las unidades regionales del Instituto Estatal de Educación para Adultos (Sicom 2006). De esta manera, se priorizó el acceso al sector educativo $\mathrm{y}$, aunque también incluían en su operación a la población en general, sólo posteriormente se estandarizaron cursos de paquetería Office para los sectores no escolarizados y adultos (Sicom y UCI Red 2006).

A partir de haber participado en distintos proyectos del Sicom entre 2003 y 2007, se pudo constatar que las principales actividades de los Centros Regionales estaban destinadas a dar cursos a las personas que no tenían acceso a tecnologías, básicamente sobre ofimática y correo electrónico. Las estadísticas de usuarios ofrecen un panorama orientado completamente hacia los estudiantes. Por señalar un ejemplo: en 2006, del total de usuarios en el telecentro localizado en la ciudad de Libres, el $78 \%$ fueron estudiantes, el $8 \%$ técnicos, el $6 \%$ amas de casa, el $4 \%$ docentes, el $2 \%$

3 El Sistema de Información y Comunicación del Estado de Puebla (Sicom) se creó en 1996 en el marco del Programa Educativo Poblado correspondiente al plan de gobierno estatal del periodo 1993-2000.

4 Además de estos CR en la Sierra Norte de Puebla, también se instalaron telecentros públicos de la misma institución en Tepeaca, Tehuacán, Libres, Ciudad Serdán y San Lorenzo Ometepec, que es junta auxiliar de Tochtepec. 
empleados y el $2 \%$ comerciantes; el $74 \%$ de ellos tenían menos de 20 años de edad (Sicom y UCI Red 2006: 16).

En recorridos realizados entre 2008 y 2011 por los Centros Regionales para documentaciones anteriores, se encontró que las personas identificaron a estos telecentros como, primero, lugares donde podían obtener una copia de la Clave Única de Registro de Población (CURP), segundo, un lugar para estudiantes, y tercero, para ir a aprender a usar la computadora ${ }^{5}$. Estos referentes los tienen casi exclusivamente los habitantes de las cabeceras municipales, porque es en ellas donde se localizan los Centros Regionales, mientras que en los demás poblados de los municipios generalmente es desconocida la existencia del telecentro o de los servicios que están disponibles.

A partir del periodo del gobierno estatal correspondiente a 2011-2017, los Centros Regionales se han convertido en centros de educación a distancia, integrando servicios de cursos virtuales de inglés y convirtiéndose en sedes para cursos vía internet de la Universidad Autónoma de Puebla, de la Universidad Nacional Autónoma de México y de la Universidad del Desarrollo del Estado de Puebla. Aunque la estrategia de operación se ha modificado, continúa la tendencia de orientar el acceso a TIC hacia la educación formal.

Los Centros Regionales sirvieron para que algunas personas aprendieran a utilizar las computadoras y para que accedieran a internet, pero, a pesar de estar abiertos a toda la población, los principales beneficiarios coincidían en dos características: habitaban en las cabeceras municipales y eran estudiantes. Si bien se han realizado iniciativas junto con los distintos gobiernos locales para incluir a personas de los pueblos aledaños, sólo han sido acciones puntuales en donde los ayuntamientos han facilitado el transporte a estudiantes de los poblados del municipio correspondiente, pero estas prácticas no se mantuvieron ni por parte del gobierno ni por las propias personas para acudir a estos telecentros y, además, terminaban centrándose en la población estudiantil. En una investigación anterior se identificó que era esporádico que las personas adultas acudieran a alguno de estos Centros, porque los vinculaban a la educación formal y porque acudir con periodicidad a los cursos resultaba más fácil para las personas que residían en el mismo poblado donde estaba localizado el telecentro que para aquellas personas que tenían que desplazarse desde otros poblados (Ramos 2009).

Los telecentros públicos inciden en la diversificación de las condiciones de conectividad y marcan diferencias entre quienes tienen o no en sus poblados un lugar de acceso público que oriente la apropiación de las TIC. Puede observarse que diez Centros Regionales distribuidos en la Sierra Norte es un número reducido; sin embargo, debido a la movilidad de las personas a través del territorio serrano, circulan ideas en torno a la existencia de lugares de acceso donde ofrecen cursos para orientar el manejo de las computadoras y que además son gratuitos o de costos reducidos. Por ello, autoridades y habitantes vislumbran ventajas si estuvieran en sus poblados: las autoridades locales los ven como parte de un progreso que los gobiernos pueden ofrecer a la población y entre los habitantes predomina la idea de que la posibilidad del acceso a TIC mejoraría la educación tanto en las aulas como fuera de ellas. En

El término «computadora» se utiliza en México, mientras que en España se utiliza más «ordenador». Por ser un estudio localizado en México, a lo largo del texto se utilizará el primero. 
este sentido, se considera a los telecentros públicos como un servicio deseable para que las generaciones jóvenes tengan la posibilidad de conexión.

Una observación final con relación a este tipo de telecentro es que su operación (mantenimiento y equipo) depende de los presupuestos del gasto público, por lo que, si bien en distintos momentos se ha cambiado el equipamiento inicial de cada Centro Regional, es evidente que se mantienen con una tecnología funcional pero obsoleta en comparación con la oferta actualizada del mercado. Aunque en años anteriores fueron los primeros lugares de acceso público a TIC en la Sierra Norte de Puebla, actualmente estos telecentros dejan de estar entre los referentes tecnológicos en la zona.

\subsection{Una propuesta nacional de telecentros públicos}

El Sistema Nacional e-México se inició en 2001 y, dentro de este programa de acceso a TIC, surgieron los Centros Comunitarios Digitales (CCD), de los cuales se desprende un tipo más específico: los Centros Comunitarios de Aprendizaje (CCA). Ambos son telecentros públicos y están distribuidos en algunas poblaciones de la Sierra Norte.

Este programa nacional estuvo compuesto por tres ejes: la conexión, los contenidos y los sistemas (SCT 2001: 256). Bajo el elemento de la «conexión» se propuso instalar lugares de acceso público a computadoras conectadas a internet, es decir, telecentros públicos. En los «contenidos» se fue incluyendo información y trámites para la ciudadanía, así como cursos de diferentes ámbitos, desde el manejo de la computadora hasta algunos más relacionados con oficios laborales. En el elemento de «sistemas» se generaron plataformas desde las cuales acceder a los contenidos, por ejemplo, la página web de los $\mathrm{CCA}^{6}$.

El programa e-México fijó tres fases para extender la red de telecentros: una primera pretendía instalar al menos un CCD en cada cabecera municipal del país, unas 2.443; después, el objetivo era cubrir cerca de 10.000 poblados donde habitaba el 75\% de la población; una tercera fase implicaría continuar ampliando la red (Pérez Salazar 2004: 130). Aunque no se cumplieron los números, este programa fue la primera propuesta a nivel nacional centrada en la conexión a internet; además, cubrió poblaciones que de otra manera no hubieran tenido acceso a tecnologías e internet sino hasta años recientes, cuando se han extendido los espectros de señales y frecuencias y han surgido más cibercafés, como ha sucedido en la Sierra Norte de Puebla.

La diferencia básica entre los CCD y los CCA es que los primeros son el prototipo amplio de telecentros públicos del programa. Posteriormente se les asignaron diferentes funciones, pues los CCA se concibieron para una atención específica hacia población con «alta» y «muy alta» marginación -categorías en donde entran la mayoría de poblaciones indígenas- siguiendo los parámetros del Consejo Nacional de Población. Se puede observar que hay aspectos diferenciadores vinculados a los lineamientos para la instalación de estos lugares de acceso; corresponde a las gestio-

\footnotetext{
${ }^{6}$ La página web es http://www.centroscomunitariosdeaprendizaje.org.mx/ desde la cual se puede acceder a los contenidos del modelo Centro Virtual de Aprendizaje, que idealmente se combina con el acceso desde los telecentros públicos, aunque se puede acceder desde cualquier lugar con conexión a internet.
} 
nes administrativas de los ayuntamientos tanto solicitar su construcción como darles mantenimiento.

Aunque este tipo de telecentros públicos comparte la misma base de actividades y servicios, han conformado diferentes procesos sociales según las interacciones que se generan entre la población. A continuación, se describen dos casos que corresponden a los municipios de Ixtepec y de Xochitlán de Vicente Suárez; en ambos se instalaron CCA y fueron los primeros lugares donde las personas pudieron acceder a las TIC.

En Ixtepec ${ }^{7}$-con población totonaca- el CCA comparte espacio con la biblioteca municipal; es una habitación de unos $50 \mathrm{~m}^{2}$. La entrada se encuentra en el costado izquierdo del edificio de la presidencia municipal; la habitación es pequeña y aun así parece tener más capacidad; la mayor parte está ocupada por los estantes en donde están depositados los libros, uno de los cuales sirve para dividir la habitación y delimitar ambos ambientes. El lado del CCA está compuesto por seis computadoras colocadas sobre escritorios con sus sillas correspondientes; estos equipos son lo primero que se ve al entrar, mientras que ya en el interior se ve una mesa al fondo en donde están amontonados otros seis equipos antiguos. Idealmente, el CCA debe contar con al menos una persona que se encargue de las actividades, pero en el año 2012, con el cambio de gobierno local, había una sola persona para atender la biblioteca y a la vez el CCA. Además, las computadoras no se podían encender porque carecían de servicio de luz suficiente para que los equipos se utilizaran.

A pesar de las dificultades circunstanciales de operación del telecentro público, las y los jóvenes en Ixtepec reconocían haber aprendido a utilizar la computadora en el CCA, donde habían seguido algunos cursos y al que habían acudido mientras fue funcional para realizar tareas escolares. Para complementar la imagen del telecentro, a continuación se transcriben algunos fragmentos del testimonio de Ana, quien fuera la primera promotora del CCA, desde 2003 hasta 2011:

«Se instaló en mayo del 2003 [...] Al inicio no teníamos cursos porque no teníamos internet, sólo el uso de la computadora, cursos de paquetería de Office, al principio fueron muchas personas las que vinieron, para los niños fue algo muy novedoso. Todas las tardes desde las 2 de la tarde estaba lleno, y para los niños estar frente a la computadora era lo bonito. A los niños les gustaba hacer dibujos y esas cosas, pero a los mayores les gustaba más hacer textos. Desde que yo estuve siempre hubo gente que acudía al CCA, en parte porque ahí no se cobra como en un ciber sino lo mínimo, bueno, primero estuvimos nosotros [CCA] y después vinieron los cibers [...].

En el 2003 nos dieron unas máquinas grandotas, eran 6 máquinas, pero después por logros del CCA en el programa nos dieron otros 6 equipos nuevos, con esos sí se trabajaba bien.

Sin internet estuvimos un año [...] ya cuando tuvimos eso fue lo bonito porque se impartían los cursos en línea, los del Tec de Monterrey [...] cursos como el de habilidades básicas en informática, creación de páginas web, el uso de internet para niños, pero en línea por medio de la plataforma del Tec; nosotros como promotores administrábamos los recursos y los servicios en línea, les ayudaba a las personas pero quien toma un curso va a su ritmo siguiendo las actividades» (Ana, Ixtepec, $11 \mathrm{de}$ julio de 2012).

\footnotetext{
7 En el municipio hay dos CCA, uno localizado en la cabecera municipal al que se hace referencia, y el otro en la junta auxiliar de San Martín, que fue instalado con posterioridad al trabajo de campo.
} 
El CCA del municipio de Xochitlán se localiza en la junta auxiliar de Huahuaxtla. En Huahuaxtla -con población mayoritariamente nahua- el telecentro tiene sus propias instalaciones, en un edificio de un piso colindante con la presidencia de la junta auxiliar. A diferencia del de Ixtepec, el de Huahuaxtla tiene la fachada pintada y los letreros correspondientes al CCA. La habitación del CCA es de aproximadamente $40 \mathrm{~m}^{2}$; los escritorios y las computadoras rodean la habitación, las pantallas quedan a la vista y quienes ocupan equipos contiguos pueden asomarse a las otras pantallas para ver lo que realizan sus compañeros y compañeras; cuenta con siete computadoras recientes más otros seis equipos proporcionados por el programa e-México. El promotor correspondiente al año 2012 había dispuesto unas 16 sillas en el centro de la habitación de modo que los asistentes se pudieran sentar para recibir cursos por medio de la señal de Edusat - para lo cual se cuenta con un televisor y material educativo- o también para la realización de cursos o pláticas - usando un proyector que se adquirió con cooperaciones económicas de la población y de la presidencia auxiliar-. En las paredes están pegados y colgados los reconocimientos al CCA por parte del Tecnológico de Monterrey y del programa e-México, por los logros alcanzados, $\mathrm{y}$, más importante, fotografías de las distintas actividades que se han realizado en el telecentro a manera de un registro gráfico.

A continuación, se citan dos testimonios de las personas que han sido promotoras del telecentro público y exponen el funcionamiento de estos lugares y su relación con la población. La primera fue Gloria, quien menciona detalles de la instalación del telecentro y algunos problemas que tuvieron en su mantenimiento y suscitaron el apoyo de la población hacia las actividades que se realizaban en él:

«Al CCA llegué en el 2003 y estuve hasta el 2008, me buscaron porque había estudiado la ingeniería informática en Zacapoaxtla, fui de la primera generación, y entonces me ofrecieron trabajo aquí en el pueblo [...] Fuimos el tercero o cuarto que se instaló en la región [...] En el pueblo no había internet, había quienes sí tenían computadoras pero no internet [...] Al inicio me preocupaba que no fuera gente a los cursos o cómo se recibirían las tecnologías, y realicé invitaciones en las escuelas para darle promoción al telecentro, pero ya había respuesta por parte de los niños porque fueron los primeros en anotarse a los cursos [...] Después me fui con los jóvenes porque sí venían muchos los niños pero me preguntaba por la demás población, hice un acuerdo con el bachillerato porque no tenían las instalaciones [...] Después los que llegaron al telecentro fueron los profesores de educación indígena porque estaban en los programas de calidad y se los exigían, y de vez en cuando alguno de educación media superior [...] A las personas mayores les costaba mucho acercarse a los equipos, hasta para manejar el mouse a veces tenía que tomarles la mano para guiarlos y se familiarizaran, en cambio para los niños era lo más fácil [...] Hubo una etapa en donde estuvimos Gil y yo, cada uno cubría un medio tiempo, pero como dependíamos de la presidencia [municipal] se cerró el CCA porque decían que ya no había dinero, pero la gente del municipio se reunió por diferentes asuntos y hasta cerraron la presidencia para que se cambiara de autoridades, y los de acá entre todo también pedían que se abriera el CCA y que regresáramos nosotros» (Gloria, Huahuaxtla, 8 de agosto de 2012).

El segundo testimonio es de Gil, quien era responsable del CCA de Huahuaxtla en 2012, y comenta algunas de las actividades que realizó y lo que se ofrece a la población desde el telecentro:

«Yo comencé a colaborar en el CCA desde el 2004 pero ya como promotor a cargo del telecentro desde el 2008 [...] soy técnico en computación pero gracias al CCA tengo 
secundaria y bachillerato [...] Hace un año [2011] tuvimos un cambio porque se optó por comprar cuatro equipos con las cooperaciones de los usuarios que vienen [...] Este telecentro ha tenido logros a nivel nacional, por eso la institución te apoya y por eso es que también tenemos otros equipos más recientes [...] Platicando con el comité [del CCA], en las reuniones, les comento que no puedo aumentar la cuota de recuperación porque tenemos competencia con los cibercafés, por eso es que mantenemos bajos precios que son para mantenimiento y para materiales del propio telecentro.

Como promotor intento facilitar los cursos que las personas vienen a tomar, por ejemplo les presento la propuesta, les comento cómo sería el proceso de aprendizaje o les pregunto si alguna vez han utilizado una computadora, o en otras ocasiones ubico a los usuarios según los cursos que ya han tomado anteriormente [...] Organizo los horarios de los grupos dependiendo de los cursos que se ofrecen, y de los grupos porque hay chicos de localidades cercanas que entre semana se quedan a vivir en Huahuaxtla porque estudian aquí, pero hay otros que estudian en sus localidades y entonces pueden venir sólo sábado o domingo y en esos días también se dispone el espacio del CCA [...] Una actividad extra que implemento es el mantenimiento y montura de equipos, y utilizamos precisamente los equipos viejos que tenemos y que son con los que comenzó el CCA» (Gil, Huahuaxtla, 16 de julio de 2012).

La instalación de este tipo de lugares de acceso público a TIC proviene de un programa nacional, pero su mantenimiento depende de la apropiación por parte de la población de las tecnologías ofrecidas, aunque más importantes son las relaciones que se establecen con las personas facilitadoras del acceso a las TIC.

En los casos de Ixtepec y Huahuaxtla se observa que las personas que son «promotoras» tienen un papel importante, porque son quienes dinamizan el acceso y quienes se convierten en los primeros contactos con las tecnologías; también realizan el seguimiento de algunas de las prácticas de internet, como vincular la conectividad con la educación. Sin embargo, las dinámicas de la población y sus apropiaciones de los telecentros son distintas: se puede observar que en Ixtepec las personas no exigieron que el servicio del CCA se renovara cuando dejó de funcionar en 2011, mientras que eso sí ocurrió en 2005 en Huahuaxtla cuando la población solicitó que se mantuviera el telecentro.

Por otro lado, los gobiernos locales también inciden en la conectividad, como se puede ver en los casos de este tipo de telecentro público, porque la manera en que está organizado el programa hace que sean los ayuntamientos los que deban cubrir los salarios de las personas promotoras, así como gestionar y cubrir los servicios para su operación, como la electricidad y el propio servicio de internet. Es decir, se genera una serie de cruzamientos en torno a la conectividad que ponen en evidencia que los gobiernos locales también tienen responsabilidades a la hora de ofrecer o mantener la posibilidad del acceso a TIC y la conexión a internet.

En pláticas ocasionales con jóvenes de Ixtepec y Huahuaxtla, éstos refirieron que habían comenzado a utilizar las computadoras con los cursos de los Centros Comunitarios de Aprendizaje y que todavía acuden a estos telecentros -lo hacían antes en el caso de Ixtepec- para la realización de tareas porque el costo es menor que el de los cibercafés, pero también fueron indicando que prefieren los cibercafés porque tienen mejores equipos y mayor ancho de banda. 


\subsection{Cibercafés}

Haciendo una comparación entre los estudios realizados por la Asociación de Internet. $\mathrm{Mx}^{8}$, se observa que entre 2009 y 2010 el acceso a internet se realizaba en primer lugar en los hogares, seguido por sitios públicos ${ }^{9} \mathrm{y}$ en tercer lugar desde el trabajo (AiMx 2011). Sin embargo, en 2014 las posiciones se alteraron: en el primer lugar se mantuvo el hogar, pero el segundo fue el trabajo, seguido por la escuela, en el cuarto lugar aparecieron los dispositivos móviles y en el quinto el acceso desde un sitio público (AiMx 2014). A nivel nacional, acudir a un sitio público como un cibercafé se redujo, al mismo tiempo que se incrementaron otros tipos de conexión. Pero actualmente, como se ha señalado en párrafos anteriores, en la Sierra Norte los cibercafés son los principales lugares de acceso a internet.

Según la Secretaría de Economía ${ }^{10}$-regulador de este tipo de comercio- un cibercafé ofrece conexión a internet y alquiler de computadoras con paquetería -que puede incluir videojuegos- $\mathrm{y}$, de manera opcional, venta de equipos informáticos y software así como artículos de papelería. Por su parte, Érika Rueda, en un estudio de cibercafés en México, define estos lugares de acceso público a TIC como «aquellos lugares donde se alquilan computadoras conectadas a la red por un precio fijo por hora, además de otros servicios» (Rueda 2008: 121); agrega también que, al ser iniciativas privadas, las características que adquieren corresponden a variables económicas y a las preferencias y conocimientos técnicos de sus propietarios, por lo que pueden encontrarse algunos espacios multimedia o simplemente básicos.

A partir de comparaciones temporales, con base en diferentes periodos de campo realizados en la Sierra Norte, se puede señalar que los costos del alquiler de equipo/ tiempo se han reducido en comparación con años anteriores, al mismo tiempo que los equipos se han ido renovando y el ancho de banda es más amplio. Aun así, se pueden encontrar lugares como la enclavada cabecera municipal de Naupan -con población nahua-, que en 2012 contaba sólo con dos cibercafés y en ambos la conexión era lenta. Un ejemplo contrario se observa en la ciudad de Huauchinango -ciudad regional y mestiza- donde las prácticas de internet son más diversificadas que el simple consumo de ancho de banda, ya que en dicha ciudad cuentan con suficiente infraestructura de telecomunicaciones, hay compañías que ofrecen el servicio de internet en las casas y también hay distintos tipos de cibercafés que van desde los que tienen veinte equipos actualizados hasta otros ubicados en los barrios circundantes que consisten en tres o cuatro computadoras instaladas en espacios improvisados, además de que cada uno de estos lugares propiciaba entornos

\footnotetext{
La Asociación de Internet .Mx utiliza datos estadísticos que generan los conteos y censos del Consejo Nacional de Población (Conapo), del Instituto Nacional de Estadística y Geografía (Inegi), la Comisión Federal de Telecomunicaciones (Cofetel), y conteos propios por medio del departamento de investigación online de la empresa Elogia. En su mayoría son datos nacionales. Recopilan la información relacionada con tecnologías de información y comunicación, como la disponibilidad de tecnología en las viviendas o el número de usuarios según los lugares de acceso, además del desglose por sexo y edad. Tienen desgloses nacionales de conexiones por días de la semana, dispositivos usados para conectarse, tiempo promedio de conexión, principales actividades online divididas en «sociales» $\mathrm{y}$ «entretenimiento» y redes sociales usadas.

9 Para un resumen de los antecedentes mundiales de los cibercafés puede verse la revisión que realizó Érika Rueda (2008: 116-118). Para un estudio breve sobre los servicios en los cibercafés puede verse el realizado en 2011 por la Procuraduría Federal del Consumidor, en http://www.profeco.gob.mx/encuesta/brujula/bruj_2011/bol189_cibercafe.asp.

${ }^{10}$ Para una guía sobre el negocio cibercafé o café internet, véase http://www.contactopyme.gob.mx/guiasempresariales/ guias.asp?s $=8 \&$ guia $=56 \&$ giro $=3$.
} 
de socialización (Ramos 2011). Es decir, el aumento de oferta y demanda de estos lugares de acceso público da lugar a que los cibercafés se incluyan como espacios sociales.

Por señalar un ejemplo más del acceso a TIC en la Sierra Norte, en la cabecera municipal de Pahuatlán -con población mayoritariamente mestiza- también es común observar que las personas acuden a los cibercafés. En 2011, un joven proveniente de un poblado nahua del mismo municipio estaba trabajando en un cibercafé y, al preguntarle por qué no trabajaba en lo mismo en Atla -su pueblo de procedencia-, respondió que la conexión a internet aún no llegaba. En contraste, en San Pablito -con población otomí-, del mismo municipio, se identificó que había cibercafés con equipos recientes y con buena conexión. En este sentido, la geografía y la infraestructura pueden influir en las posibilidades de conexión a internet, pero no son determinantes: en los casos de los poblados nahua y otomí de Pahuatlán, los aspectos determinantes eran los recursos económicos y la capacidad de gestión que los otomíes han desplegado para obtener distintos servicios.

En los casos de Ixtepec, Huahuaxtla y Xochitlán, había cibercafés con buen ancho de banda -aunque en ellos se indicaba que no se podían ver videos porque reducían la velocidad de los demás equipos, una situación que estaba cambiando al comenzar a contratarse servicios sin límite de descarga- y un flujo considerable de personas, generalmente en las tardes, porque en su mayoría son las y los jóvenes quienes acuden después del horario escolar. Los habitantes de estos poblados identifican de manera fácil los cibercafés, conocen los servicios que se ofrecen y son sitios localizables para ellos. Un extracto del diario de campo ilustra esta situación:

«A veces sólo por sondear si las personas tienen ubicados los cibercafés pregunto a quienes van por la calle si saben de algún ciber cercano [...]. En los tres pueblos en donde he estado y hasta en los poblados previos [...] me indicaron a dónde podía acudir, también preguntaba si sabrían si tenían conexión o sólo eran lugares para alquilar un ordenador, o si podría sacar fotocopias o imprimir, etcétera, y me sirvió mucho ese tipo de sondeo ocasional para identificar que a excepción de algunas personas adultas [...] no sólo tienen identificados los cibercafés sino también los servicios que pueden obtener en cada uno de ellos» (Diario de campo, 17 de julio de 2012).

Los cibercafés permiten, coincidiendo en esto con Érika Rueda (2008: 164-165), que las personas sin posibilidades económicas de cubrir el costo de un equipo y de la conexión mensual a internet -en las áreas donde sí hay cobertura- puedan ir pagando las pequeñas cuotas del acceso a TIC a través de los cibercafés; constituyen lo que la autora llama «modelo de conectividad popular» (2008: 149). Diferimos, sin embargo, de Rueda cuando señala que las incursiones que realizan las personas por medio de los cibercafés hacen que se incorporen a la «Sociedad de la Información» (2008: 225).

Retomando la noción de campo social de William Roseberry (1998), la conectividad en la Sierra Norte de Puebla implica que los diferentes lugares de acceso público a TIC -además de las conexiones que las y los usuarios realizan desde sus perfiles de redes sociales- se han convertido en espacios que generan y posibilitan relaciones sociales específicas en torno a las tecnologías digitales y a internet. Este aspecto se considera relevante porque las relaciones y dinámicas locales se diversifican y hacen más complejas a partir de estos lugares; no sustituyen ningún otro espacio social ni restan posiciones a otros temas que emergen y circulan en los poblados indígenas, sino que se colocan dentro de los entramados de significación local en los que los su- 
jetos sociales se acomodan, responden o enfrentan. En la exposición de este asunto, a través de personas concretas, se centra el siguiente apartado.

\section{Las personas nodos}

Las personas facilitadoras que guían el acceso y uso de las tecnologías digitales y de internet adquieren importancia porque en muchas ocasiones son quienes orientan los primeros acercamientos a las TIC, también porque viven entre la población y tienen una posición en las comunidades; son -junto con sus cualidades personales- puentes entre el campo de la conectividad y otros espacios de la vida local. En este sentido, se constituyen como nodos entre las personas y las tecnologías.

Caracterizar a estas personas-nodos realizando una clasificación de ellas resultaría insuficiente, porque, así como cada lugar de acceso público a TIC tiene diferentes características, cada persona situada en dichos lugares también presenta distintas facetas. Los siguientes párrafos se aproximan a esa caracterización mediante la yuxtaposición de casos de personas concretas - diferentes entre sí- que permiten apreciar su incidencia en las prácticas en torno a las tecnologías digitales e internet y las cualidades de sus interacciones con la población.

En la cabecera municipal de Xochitlán funcionaban dos cibercafés, pero los padres y madres de estudiantes de bachillerato lograron reunir fondos y, junto con los directivos de la escuela, acondicionaron un salón para las clases relacionadas con la informática. Un profesor, José, estaba a cargo de cuatro materias (Introducción a los sistemas operativos, Aplicaciones informáticas, Redes, y Mantenimiento correctivo) de segundo y tercer año de bachillerato. La mayoría de los grupos que José ha atendido suelen estar conformados por 30 estudiantes -cada curso cambia el número de estudiantes-; el salón tiene $70 \mathrm{~m}^{2}$ aproximadamente y está dotado de 21 mesas con sus correspondientes computadoras, distribuidas por todo el espacio; dada la cantidad de computadoras, algunos estudiantes tenían que compartir equipos.

La visión de José con respecto a la informática es que hay "una simulación", porque quien realmente sabe de informática es aquel que utiliza lenguaje binario y programación, mientras que el resto se limita a interactuar por medio de programas que son interfaces. José entiende que las clases actuales centran sus contenidos en aspectos generales de las opciones para aplicar la informática, para que quienes se interesen continúen estudiando. También comentó que espera que sus alumnos puedan realizar otras actividades y que por eso les exige en las clases y en las tareas, porque conoce gente de Zacapoaxtla -ciudad regional con población mestiza- que ha salido de la ingeniería de informática y lo que hacen es abrir un cibercafé, lo cual para José significa continuar con la simulación de la interacción con las computadoras -por medio de la reproducción del uso de programas interfaces-. Comenta que sabe que en la región no hay demanda para ese tipo de carreras, pero que, al mismo tiempo, son una oportunidad para generar ideas (Diario de campo, Xochitlán, 29 de mayo de 2012). Pero si bien José cumplía con sus clases y labores, él viajaba todos los días tres horas ida y vuelta desde Zaragoza -poblado mestizo-; no estaba completamente involucrado con las y los estudiantes o con las actividades y problemáticas de la población.

En Ixtepec, el telecentro público estuvo en servicio desde 2003 hasta 2011 y durante ese tiempo estuvo a cargo de la misma persona, Ana. Ella estudió la ingeniería 
informática en Zacapoxtla -de la segunda generación del Instituto Tecnológico Superior de Zacapoaxtla-y también una licenciatura en administración de empresas, ya siendo promotora y acomodando sus tiempos para que los sábados pudiera ir de su pueblo a la ciudad regional. Cuenta que salió del CCA porque, dependiendo el telecentro de la presidencia municipal, las autoridades que llegaron a la misma en 2011 prefirieron colocar a las personas que les apoyaron en la campaña electoral; si hubiera dependido de su decisión, habría preferido continuar como promotora, porque considera que es una labor social para su comunidad. Cuando estuvo a cargo del telecentro comentó:

«Es importante que los jóvenes y los niños se asomen a este mundo, porque adquieren otros conocimientos que muchas generaciones, como la mía, no tuvimos en nuestro pueblo. Sin duda, los niños y jóvenes deben conocer la importancia del avance de la tecnología. Por ejemplo, en mi caso, tuve el primer contacto con una computadora hasta la carrera universitaria. Entonces esto es una gran ventaja» (Yanes 2006: 93).

Después del CCA, Ana abrió un cibercafé. Era una habitación de menos de $40 \mathrm{~m}^{2}$, con 12 computadoras, una impresora y escáner; además ella estaba en una mesa con su propia computadora y desde ahí controlaba los tiempos de alquiler. Por su experiencia en el telecentro público, y como habitante del poblado, sabía que eran constantes las interrupciones de electricidad -en algunas temporadas del año son constantes las tormentas- por lo que tenía un suministro auxiliar que le daba tiempo suficiente para apagar los equipos sin que se dañaran; además, a las computadoras les había instalado un sistema de bloqueo o congelamiento, con lo cual les daba libertad a las personas usuarias para que descargaran programas y archivos sin que afectaran el equipo, porque cada determinado tiempo regresaban al estado del sistema en el que se habían congelado.

En una conversación con ella, Ana relató que cuando estaba trabajando en el CCA sus amistades le preguntaban si se aburría en ese sitio o si se desesperaba por tener que lidiar con las niñas y los niños. Afirma que se considera una persona paciente y que por eso no se aburría; al contrario, regresaría si se diera la ocasión. También comentó que, al ser una persona conocida en el pueblo, tuvo relación con casi todas las personas, las que llevaban a sus hijas o hijos, las que acudían para que les buscara alguna información en internet o algo técnico; por eso dice que, si aún la buscan, es porque saben quién es la persona y no por lo que se tiene materialmente (Diario de campo, Ixtepec, 11 de julio de 2012).

En Huahuaxtla, el telecentro público ha tenido dos promotores. La primera fue Gloria, a quien las autoridades locales buscaron para ofrecerle el puesto en el telecentro porque la consideraron la persona adecuada para hacerse cargo del espacio, ya que estaba relacionada con "aquello de las computadoras". Ella cuenta que desde la apertura del CCA hubo mucha demanda de los niños y niñas, y de las y los jóvenes, por lo que al año de la apertura solicitó al presidente auxiliar que contrataran a otra persona; así es como se incorporó uno de los jóvenes que mostraban más participación en las actividades del telecentro, Gil, y durante cuatro años ambos compartieron responsabilidades. En 2005 se vivió un momento de tensión política en el municipio que desembocó en el cierre del telecentro, pero entre las demandas que la población hacía a las autoridades locales se incluyó el que se reabriera el lugar en donde las generaciones jóvenes recibían cursos y apoyaban los procesos educativos formales. 
En 2008 Gil se quedó a cargo del CCA y desde entonces ha aprovechado que el programa e-México aliente a los promotores para que realicen actividades complementarias dependiendo de las coyunturas y de las necesidades de las poblaciones. Un ejemplo son las «pláticas» que Gil ha organizado entre jóvenes que han salido y están en las ciudades y jóvenes que están por salir del pueblo. La idea surgió después de observar que los jóvenes que salen hacia las ciudades van olvidando «las raíces» y cuando regresan después de algunos años ya no quieren hablar en nahua o mantienen actitudes de distancia frente a las costumbres y tradiciones locales. Por ello pensó en una actividad que mantuviera una idea de comunidad entre los jóvenes; en sus propias palabras: «son quienes están por salir del pueblo y está bien que quieran regresar al pueblo, pero que además aporten a la comunidad» (Diario de campo, Huahuaxtla, 21 de julio de 2012).

Lo que se pudo apreciar en el telecentro es que todos los días había grupos de niños y niñas, de jóvenes y también adultos; un sábado, al hacerle unas preguntas a un niño que estaba en el telecentro, éste dijo que provenía del poblado de Pahuata -con población nahua- y que sólo esos días podía ir a Huahuaxtla, con lo que se hacía evidente la eficacia de la distribución de horarios y días. Después de más diez años, continúa siendo un espacio dinámico, a pesar del incremento de cibercafés y de que hasta las características personales del promotor han sido puestas en valoración; pero Gil ha logrado identificar los huecos de la conectividad y ha intentado incidir para ampliar el acceso y uso de las TIC desde el telecentro público.

Como se ha podido observar en los retratos anteriores, las personas facilitadoras del acceso a las TIC tienen rasgos personales que pueden influir en las maneras con que se realizan los acercamientos y exploraciones hacia las tecnologías digitales y hacia internet. Pero estas personas, además, se encuentran inmersas en interacciones -diversas y a veces conflictivas- con las poblaciones que atienden, por lo que también comparten valores y sentidos de la comunidad, entre otras relaciones y subjetividades. Así, estas personas-nodos participan en los distintos espacios sociales a la vez que incentivan uno, aquel en el que tienen mayor control de los recursos, el vinculado a las tecnologías digitales.

\section{La constante configuración social del acceso a lo digital}

El campo social vinculado a la conectividad en los poblados indígenas se despliega desde condiciones desiguales de acceso en comparación con otros lugares que tienen mayor infraestructura y tecnologías -no sólo ciudades capitales, también ciudades regionales de la Sierra Norte de Puebla-, pero se trata, al mismo tiempo, de conectividades heterogéneas que reflejan la variedad del acceso y las apropiaciones de las TIC. En este artículo se ha colocado el foco en los lugares donde las personas pueden ir a utilizar una computadora, conectarse a internet o, en un momento previo, aprender a utilizar la computadora, y se ha subrayado el papel de las personas que se encargan de facilitar el acceso y utilización de las tecnologías digitales en estos lugares.

Un acercamiento histórico al acceso a las TIC en los poblados indígenas -por mínimo que sea, como el que aquí se ha expuesto- permite observar que estos accesos se han ido conformando a partir de la acción de distintos sujetos sociales en diferentes momentos, quienes se relacionaron y se relacionan desde las interpretaciones de sus mundos sociales, incluyendo, por ejemplo, las políticas públicas vinculadas a las 
TIC, la educación formal, las tendencias tecnológicas o la priorización de necesidades individuales, familiares y colectivas. Así, las situaciones difieren en el tiempo y continúan modificándose. Un ejemplo representativo bajo esta perspectiva puede ser lo que ha sucedido con los Centros Regionales, pues fueron los primeros lugares donde las personas pudieron utilizar una computadora, cuando lo importante era acceder a estas tecnologías -reducir la brecha digital-; actualmente se han multiplicado los lugares de acceso público a TIC y las características tecnológicas son más relevantes, por lo que este tipo de telecentro ha dejado de ser un referente, aunque, paradójicamente, lo es menos por el rezago tecnológico que por la falta de adecuación de las políticas públicas e innovación pedagógica. Mientras tanto, los cibercafés, si bien carecen de propuestas didácticas, responden a la oferta y la demanda, por lo que han ofrecido equipos actualizados y, cada vez más, mejor ancho de banda.

Por otro lado, se puede apreciar que el acceso a las TIC fue dirigiéndose, indirectamente, hacia diferentes personas. Los primeros lugares de acceso provinieron de agendas digitales por parte de los gobiernos federal y estatal, y coincidieron en emitir un discurso de apertura a toda la población; sin embargo, en la operación local se dirigieron al sector escolar, básicamente estudiantes y, después, al profesorado que -como bien señala Gloria del telecentro de Huahuaxtla- comenzó a acudir cuando se les pidió como parte de sus capacidades docentes. Esta primera imagen puede apreciarse desde dos desigualdades preexistentes, por un lado la económica, que restringía el acceso a la mayoría de tecnologías de información y comunicación, y por otro lado la educativa, pues los índices de alfabetismo aún son bajos en los poblados indígenas ${ }^{11} \mathrm{y}$, específicamente para las personas adultas, no sólo porque fuera necesario leer y escribir, sino porque tampoco estaban familiarizadas con herramientas como las TIC.

Posteriormente, el acceso ha comenzado a extenderse, en menor medida por algún cambio de estrategia en los lugares de acceso -telecentros y cibercafés-, pero sobre todo porque la población fue acercándose gradualmente, incorporando, además de los profesores, personas que interrumpieron sus años escolares -jóvenes no estudiantes-, migrantes en otras ciudades del país o en Estados Unidos, comerciantes y profesionistas oriundos de los poblados y que regresan por temporadas o de manera permanente. Así, esta segunda imagen muestra que, si bien continúa la tendencia a vincular tecnologías y educación, tal relación se está haciendo flexible y se amplía fuera de la educación formal; por otro lado, puede observarse que las y los jóvenes siguen siendo las principales personas que acceden y utilizan las TIC, pero de igual manera hay otros sectores etarios que con mayor frecuencia se relacionan por medio de las tecnologías digitales.

Además de observar las transiciones temporales, se pueden ir identificando diferentes sujetos sociales individuales y colectivos en interrelación: los gobiernos locales que han gestionado la instalación de un telecentro bajo la idea de ofrecer progreso a los pobladores; una serie de personas que han identificado que un cibercafé puede ser redituable económicamente; los padres y las madres de estudiantes organizándose junto con los directivos de las escuelas para adecuar salones con computadoras; jóvenes estudiantes y no estudiantes que acuden frecuentemente a los lugares de acceso público a TIC para hacer tareas escolares o para conectarse a internet; pobla-

\footnotetext{
${ }^{11}$ El total de la población indígena en México, con edad de 15 años y más, que no puede leer y escribir es el 23\% (Inegi 2015).
} 
dores que a pesar de no entrar en los telecentros o en los cibercafés pueden identificar servicios, usos y tecnologías que hay en esos lugares; personas-nodos a cargo de lugares de acceso a TIC que al ser parte -cuando lo son- de las comunidades están involucradas en las dinámicas locales.

Quizá estas últimas personas mencionadas sigan teniendo un papel destacable, aún con el paso del tiempo y el incremento de adquisición de tecnología por la población en general, porque son quienes median la relación inicial y referencial de las tecnologías digitales, ya que han adquirido otras capacidades que anteriormente no eran necesarias para la vida en las poblaciones rurales e indígenas y que ahora forman parte de los entramados de significación locales.

Desde los ámbitos locales se pueden mencionar aspectos inmersos en las relaciones sociales en torno al acceso y uso de las TIC. Éstos se mueven alrededor de la conformación de la comunidad, en el sentido de lo que Tesiu Rosas Xelhuantzi (2008) denomina «merecer la comunidad», es decir: entendiéndola no como algo dado, sino más bien desde la participación e involucramiento de las personas hacia aquello que consideran su comunidad. Así, las personas-nodos mencionadas ofrecen situaciones distintas y se puede observar el despliegue de diferentes relaciones según la pertenencia. Por ejemplo, algunos comentarios de Ana y Gil apuntan hacia la forma de hacer comunidad: uno es el énfasis en el servicio a la comunidad, que va más allá del mero acceso a TIC; otro punto, relacionado con el anterior, es precisamente que las personas-nodos son referentes para la resolución de problemas concretos desde los telecentros y, en casos particulares como el de Ana, desde los cibercafés; y un tercer punto son los valores colectivos que circulan localmente, con los cuales distinguen las actitudes de las personas hacia sus vecinos.

En este sentido, se considera que la conformación del acceso a las TIC en los poblados indígenas de la Sierra Norte de Puebla corresponde, además de a las desigualdades socioeconómicas y las políticas de exclusión, a las acciones, decisiones, intereses y capacidades de distintos sujetos sociales en procesos sociales e históricos específicos, muchas veces respondiendo precisamente a desigualdades y exclusiones; a su vez, estos espacios sociales en torno a las TIC se van modificando, pero manteniéndose o restableciéndose como articuladores de otras relaciones sociales, también cambiantes.

\section{Conclusiones}

Las páginas anteriores han buscado exponer las condiciones materiales y las características del acceso a las tecnologías de información y comunicación en el contexto de los pueblos indígenas, particularmente de la Sierra Norte de Puebla. Se ha partido de un hecho evidente en cualquier recorrido por los poblados indígenas: en ellos se utiliza alguna tecnología digital y los usos, así como su incorporación a diferentes actividades cotidianas, son muy diversos. Sin embargo, las condiciones de desigualdad y de exclusión hacen que el acceso a las TIC implique relaciones diferenciales entre quienes pueden acceder y quienes no, así como también entre quienes utilizan las TIC de manera frecuente. En este sentido, antes de iniciar un acercamiento a las incursiones digitales, se ha indagado en torno a las posibilidades del acceso a las TIC.

Lo que se ha encontrado es que el acceso a las TIC se había inclinado, de manera directa e indirecta, hacia la población estudiantil y que aún sigue predominando el 
vínculo entre tecnologías y educación. Esta situación está cambiando y actualmente se pueden identificar diferentes personas usuarias con distintas edades, actividades laborales y grados educativos, aunque hay otras desigualdades que sesgan el acceso para toda la población. Los telecentros públicos han tenido un papel importante en este proceso, tanto por el acercamiento de las tecnologías como por las maneras en que se insertan en las dinámicas locales; en este sentido, las personas responsables de estos lugares de acceso adquieren un papel que supera las relaciones de facilitación, ya que además incentivan un espacio social ligado a las tecnologías digitales y lo hacen desde sus propias posiciones sociales y subjetividades compartidas localmente.

A pesar de la relevancia de los telecentros, incluso en poblaciones con escasos servicios públicos, lo cierto es que están implantados sólo en algunos poblados y dependen del gasto público. En contraste, continúan apareciendo cibercafés en todos los poblados, ya sea con equipos básicos y conexión lenta o con mejores características multimedia y amplio ancho de banda, y se han convertido en los lugares predominantes para conectarse a internet. Así, se ha podido observar que, en la posibilidad del acceso a TIC, los gobiernos en sus distintos niveles tienen un papel de responsabilidad a la hora de incentivarla o limitarla a través de la dotación de servicios como electricidad e internet o de la formulación de innovaciones pedagógicas. Esta situación es compartida con las dinámicas locales, donde intervienen las relaciones que las personas establecen con las tecnologías digitales y las coyunturas que pueden motivar a la organización colectiva a demandar o gestionar servicios que se van considerando importantes.

Como cabe observar, una lectura desde los campos sociales permite identificar procesos, agencias y sujetos sociales, relaciones y contradicciones, que desde los contextos locales se vinculan a las tecnologías de información y comunicación, conformando un acceso a internet desigual y heterogéneo. Dicha lectura puede ayudar a comprender las incursiones digitales por parte de los pueblos indígenas, en particular al considerar que la «posibilidad» del acceso a internet tiene diferentes matices (en algunos poblados aún es algo a conseguir), y al subrayar que las expresiones y relaciones digitales están imbricadas con otras relaciones y mediaciones previas.

\section{Referencias}

Asociación de Internet .Mx (AiMx). 2011. «7º estudio sobre los hábitos de los internautas en México». https://www.asociaciondeinternet.mx/es/component/remository/Habitos-deInternet/Estudio-sobre-los-habitos-de-los-usuarios-de-internet-en-Mexico-2011/lang,eses/?Itemid.

_. 2014. «Estudio sobre los hábitos de los usuarios de internet en México 2014». https:// www.asociaciondeinternet.mx/es/component/remository/Habitos-de-Internet/Estudio-sobrelos-habitos-de-los-usuarios-de-internet-en-Mexico-2014/lang,es-es/?Itemid.

—. 2016. «12 ${ }^{\circ}$ estudio sobre los hábitos de los usuarios de internet en México 2016». https://www.asociaciondeinternet.mx/es/component/remository/Habitos-de-Internet/12Estudio-sobre-los-Habitos-de-los-Usuarios-de-Internet-en-Mexico-2016/lang,es-es/?Itemid.

Barbosa Cano, Manlio. 2012. Las regiones naturales, étnicas y culturales de Puebla. Puebla: Ediciones de Educación y Cultura y Facultad de Filosofía y Letras, Benemérita Universidad Autónoma de Puebla. 
Becerra Cortés, Yunuén Esperanza. 2012. «Estudiantes indígenas y los usos y apropiación de las tecnologías de información y comunicación». Paakat: Revista de Tecnología y Sociedad 3. http://www.udgvirtual.udg.mx/paakat/index.php/paakat/article/view/180/252.

Burrell, Jenna. 2011. «User Agency in the Middle Range: Rumors and the Reinvention of the Internet in Accra, Ghana». Science, Technology \& Human Values 36 (2): 139-159.

Centro de Estudios sobre Calidad de Vida (CECAVI). 2000. «Diagnóstico de los Centros Regionales». Informe técnico. Puebla: Universidad de las Américas.

Consejo Nacional de Población (CONAPO). 2015. «Índice de marginación por entidad federativa 2015». http://www.conapo.gob.mx/es/CONAPO/Datos_Abiertos_del_Indice_de_ Marginacion.

Delgado, Guillermo. 2002. «Solidarity in Cyberspace: Indigenous Peoples Online». NACLA Report on the Americas 35 (5): 49-51. https://doi.org/10.1080/10714839.2002.11724572.

Gómez Mont, Carmen. 2005. Tejiendo hilos de comunicación: los usos sociales de internet en los pueblos indígenas de México. Tesis doctoral. México: Universidad Nacional Autónoma de México.

Guerra García, Ernesto y Erasmo Miranda Bojórquez. 2010. «Apropiación de las NTIC por los indígenas yoremes-mayo», en Migrantes e indígenas: acceso a la información en comunidades virtuales interculturales, Eduardo Sandoval Forero y Ernesto Guerra García, eds., pp. 169-223. México: Universidad Autónoma del Estado de México - Universidad Autónoma Indígena de México.

Hargittai, Eszter. 2004. «Internet Access and Use in Context». New Media \& Society 6 (1): 137-143.

Instituto Nacional de Estadística y Geografía (INEGI). 2011. Principales resultados del Censo de Población y Vivienda 2010. México. México: INEGI. https://www.inegi.org.mx/ app/biblioteca/ficha.html?upc=702825002042.

. 2015. «Encuesta Intercensal, 2015» (Boletín de prensa $n^{\circ}$ 524/15). http://www.inegi. org.mx/saladeprensa/boletines/2015/especiales/especiales2015_12_3.pdf (véase también http://www.beta.inegi.org.mx/app/descarga/?ti=9).

—. 2016. «Encuesta Nacional sobre Disponibilidad y Uso de Tecnologías de la Información en los Hogares, 2015» (Boletín de prensa $n^{\circ}$ 131/16). http://www.inegi.org.mx/ saladeprensa/boletines/2016/especiales/especiales2016_03_01.pdf (véase también http:// www.beta.inegi.org.mx/app/descarga/?ti=9).

Pérez Salazar, Gabriel. 2004. Análisis crítico del Sistema Nacional e-México: La estrategia web del gobierno federal para la reducción de la brecha digital. Tesis de maestría. México: Universidad Nacional Autónoma de México.

Ramos Mancilla, Oscar. 2009. Un clic diferente. Mujeres rurales, tecnologías y cibercultura en Allende, Cuyoaco, Puebla. México: Inmujeres.

- 2011. «Conexiones digitales en una ciudad de la Sierra Norte de Puebla», en Huauchinango. El rumor del tiempo, Libertad Mora, coord., pp. 341-360. México: Gobierno Municipal de Huauchinango - Perspectivas Interdisciplinarias en Red A.C.

Rosas Xelhuantzi, Tesiu. 2008. Descolonizar el pueblo. Cuestionamientos al eurocentrismo desde la perspectiva náhuatl. Tesis doctoral. México: Universidad Nacional Autónoma de México.

Roseberry, William. 1998. «Cuestiones agrarias y campos sociales», en Las disputas por el México rural, Vol. 1: Actores y campos sociales, Sergio Zendejas y Pieter de Vries, eds., pp. 73-97. Zamora: El Colegio de Michoacán.

Rueda Ramos, Érika. 2008. Acceso público a Internet: los cibercafés en México. México: Fundación Manuel Buendía. 
Sandoval Forero, Eduardo Andrés. 2013. «Los indígenas en el ciberespacio». Agricultura, Sociedad y Desarrollo 10 (2): 235-256.

Secretaría de Comunicaciones y Transportes (SCT). 2001. «Sistema Nacional e-México», en Programa Sectorial de Comunicaciones y Transportes 2001-2006, pp. 253-271. México: Coordinación General de Planeación y Centros SCT - Dirección General de Planeación.

Sistema de información y Comunicación del Estado de Puebla (Sicom). 2006. Historia de los Centros Regionales: un caso de éxito y digno de aprender. Documento interno, Sistema de Información y Comunicación del Estado de Puebla.

Sistema de información y Comunicación del Estado de Puebla y Universidad Campesina e Indígena en Red (Sicom y UCI Red). 2006. Diagnóstico Centro Regional de Libres, Puebla, México. Documento de trabajo, Sistema de Información y Comunicación del Estado de Puebla.

Yanes Rizo, Emma, coord. 2006. Historia viva. Ixtepec. México: Instituto Nacional de Antropología e Historia - Fundación para la Investigación Social y Ambiental de México y sus Regiones S.C. 